Supporting Information

\title{
Vapor-Liquid-Solid Growth and Optoelectronics of Gallium Sulfide van der Waals Nanowires
}

\author{
Eli Sutter,,$^{1, *}$ Jacob S. French, ${ }^{2}$ Stephan Sutter, ${ }^{2}$ Juan Carlos Idrobo, ${ }^{3}$ and Peter Sutter ${ }^{2, *}$ \\ ${ }^{1}$ Department of Mechanical \& Materials Engineering, University of Nebraska-Lincoln, Lincoln, \\ Nebraska 68588, USA \\ E-mail: esutter@unl.edu \\ ${ }^{2}$ Department of Electrical \& Computer Engineering, University of Nebraska-Lincoln, Lincoln, \\ Nebraska 68588, USA \\ E-mail: psutter@unl.edu \\ ${ }^{3}$ Center for Nanophase Materials Sciences, Oak Ridge National Laboratory, Oak Ridge, TN 37831, \\ United States
}

\section{Supporting Figures}
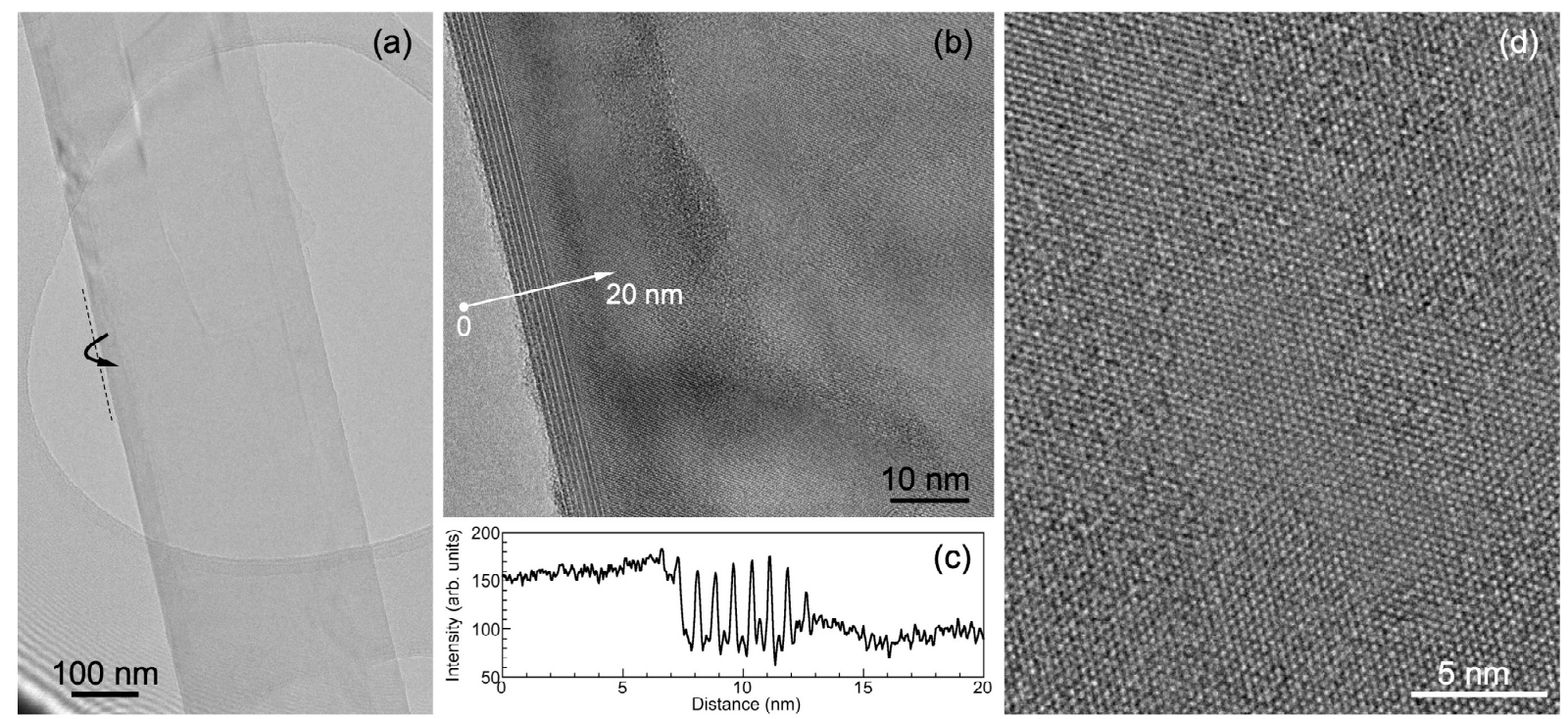

Figure S1. Ultrathin ribbon-like type (i) GaS nanowires. (a) TEM image of a type (i) GaS nanowire with folded edge, as indicated (arrow). (b) High-resolution TEM image near the edge, showing the GaS layering along the folded region. (c) Intensity line profile along the arrow marked in (b) showing 8 intensity maxima, i.e., a thickness of $8 \mathrm{GaS}$ monolayers. (d) High-resolution TEM image obtained within the nanowire, showing the hexagonal structure of basal-plane oriented GaS (zone axis, ZA: [0001]). 

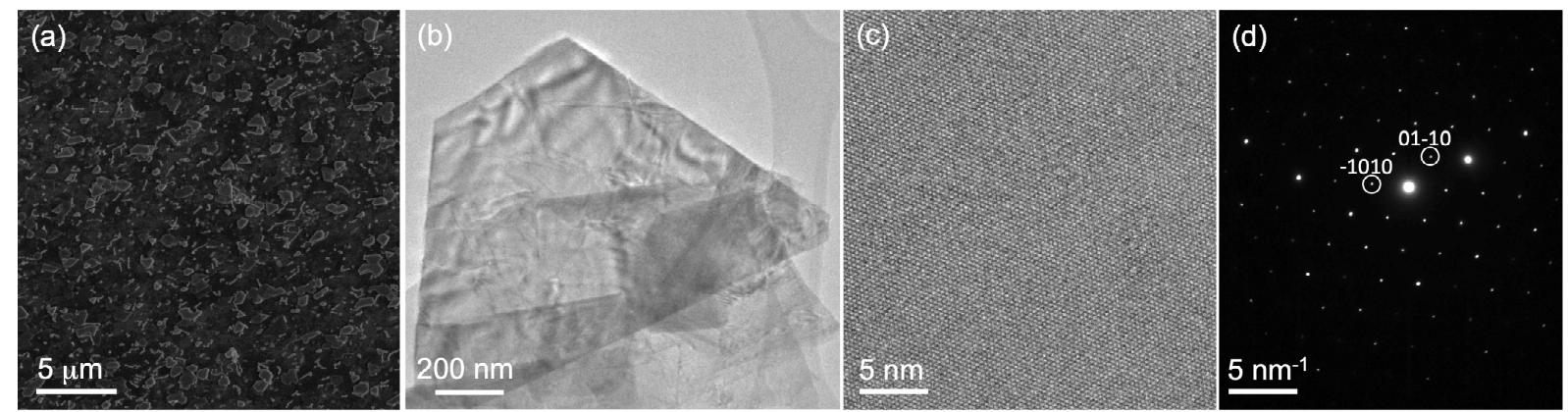

Figure S2. GaS flakes grown on Au catalyst on $\mathrm{SiO}_{2}$. (a) SEM image of a section of an as-grown $\mathrm{GaS}$ on $\mathrm{SiO}_{2} / \mathrm{Si}$ covered with a thin Au film. (b) Typical GaS flakes dry transferred to a carbon TEM grid. (c) High-resolution TEM image, showing the characteristic hexagonal structure of basal-plane oriented GaS. (d) Selected-area electron diffraction pattern (zone axis, ZA: [0001]) of the flake.
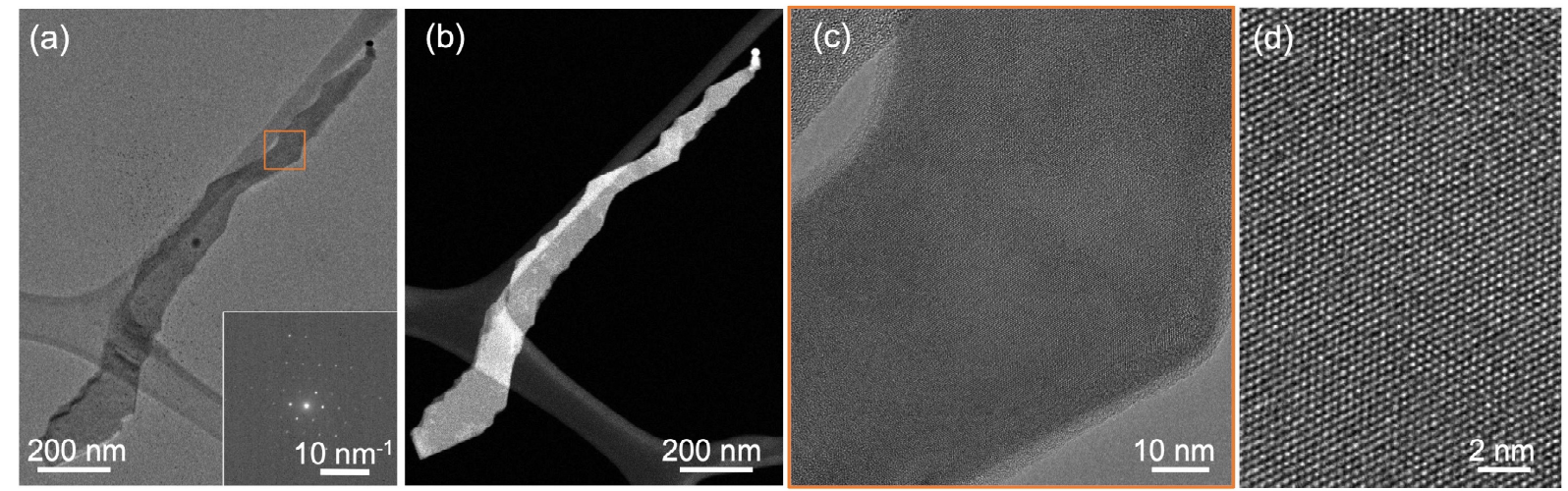

Figure S3. Ribbon-like type (i) $\mathrm{GaS}$ nanowires grown with Au catalysts on $\mathrm{SiO}_{2}$. (a) $\mathrm{TEM}^{\mathrm{T}}$ image of a ribbon-like GaS nanowire synthesized by VLS growth with Au catalyst. Note the small Au-rich particle at the tip (upper right). Inset: Selected-area electron diffraction pattern (Zone axis, ZA: 0001). (b) HAADF-STEM image of the GaS nanowire shown in (a). (c) Magnified view of the area marked by a rectangle in (a). (d) High-resolution TEM image of the basal-plane GaS lattice structure.

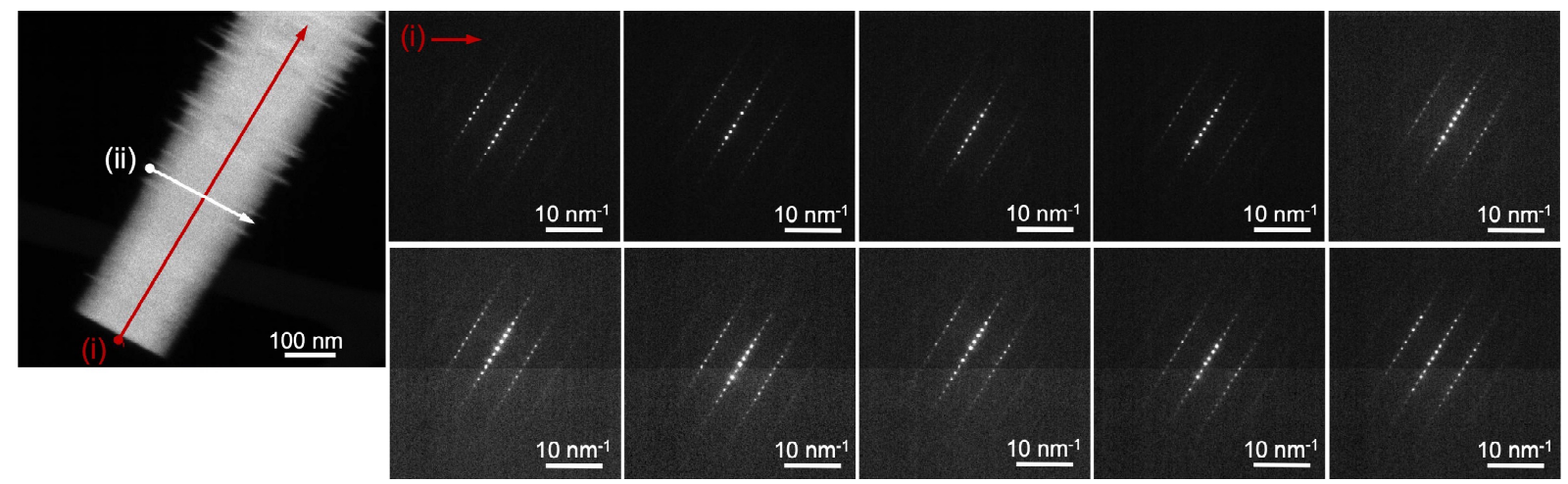

Figure S4. Nanobeam diffraction along a tapered type (ii) GaS nanowire. (a) HAADF-STEM image obtained near the thinner end of the nanowire. (b) Nanobeam diffraction patterns obtained along the nanowire (line marked (i) in panel (a)). The series comprises every $5^{\text {th }}$ diffraction pattern of a total of 50 patterns acquired along the line (i) shown in (a). 


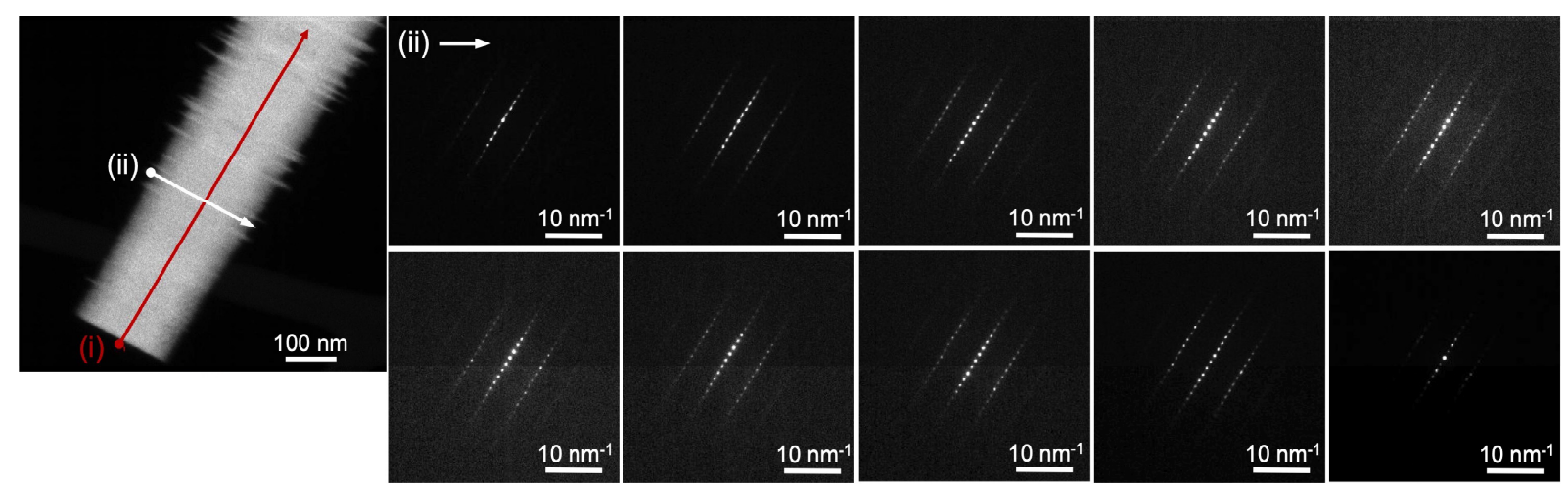

Figure S5. Nanobeam diffraction across a tapered type (ii) GaS nanowire. (a) HAADF-STEM image obtained near the thinner end of the nanowire. (b) Nanobeam diffraction patterns obtained across the nanowire (line marked (ii) in panel (a)). The series comprises every $3^{\text {rd }}$ diffraction pattern of a total of 30 patterns acquired along the line (ii) shown in (a).

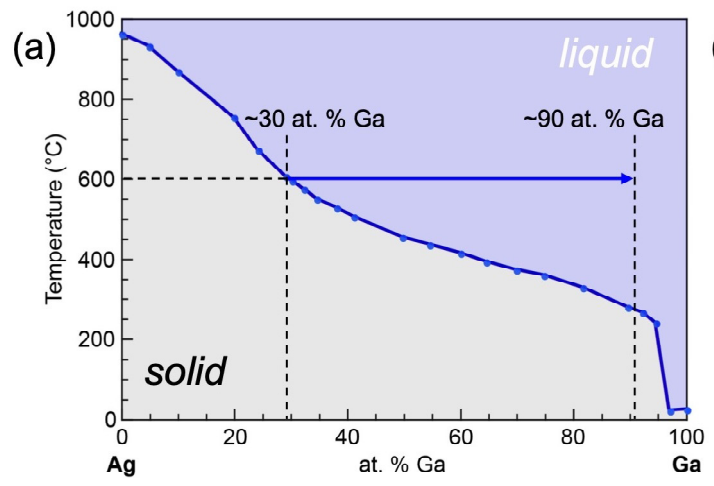

(d)

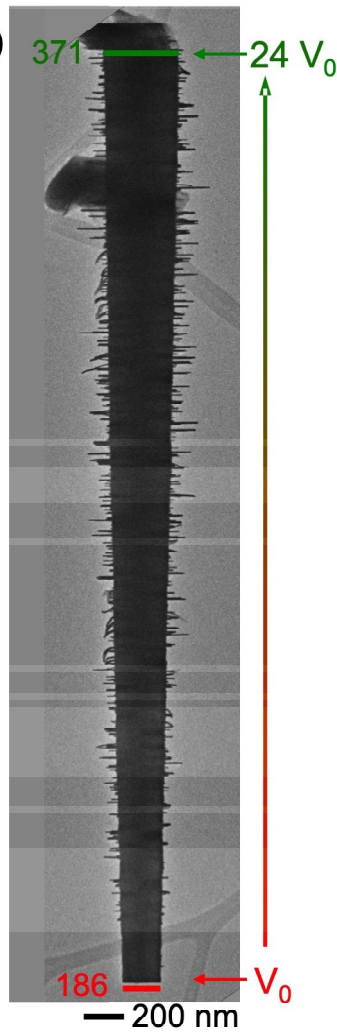

Figure S6. Estimation of the size evolution of the VLS catalyst during growth of tapered type (ii) GaS nanowires. (a) Partial Ag-Ga phase diagram, showing the composition-dependent liquidus line of the binary alloy. Note: Growth temperature in our experiments is $600^{\circ} \mathrm{C}$. Arrow: Evolution of the $\mathrm{Ga}$ content from an initial catalyst with composition that corresponds to a melting temperature of $600^{\circ} \mathrm{C}$. (b) SEM image of two type (ii) GaS nanowires, grown with Ag catalysts. Note the catalyst particles at their tips. (c) Analysis of the spherical-cap shape of the solid catalyst. (d) TEM image of a type (ii) GaS nanowire, showing the diameters at the root $(186 \mathrm{~nm})$ and tip $(371 \mathrm{~nm})$. Estimating the shape of the molten catalyst as equal to that after crystallization (as shown in (b)), the tapered nanowire shape implies a 24-fold increase of the catalyst volume between the early (volume $V_{0}$ ) and final stages (volume $24 \times V_{0}$ ) of growth. 

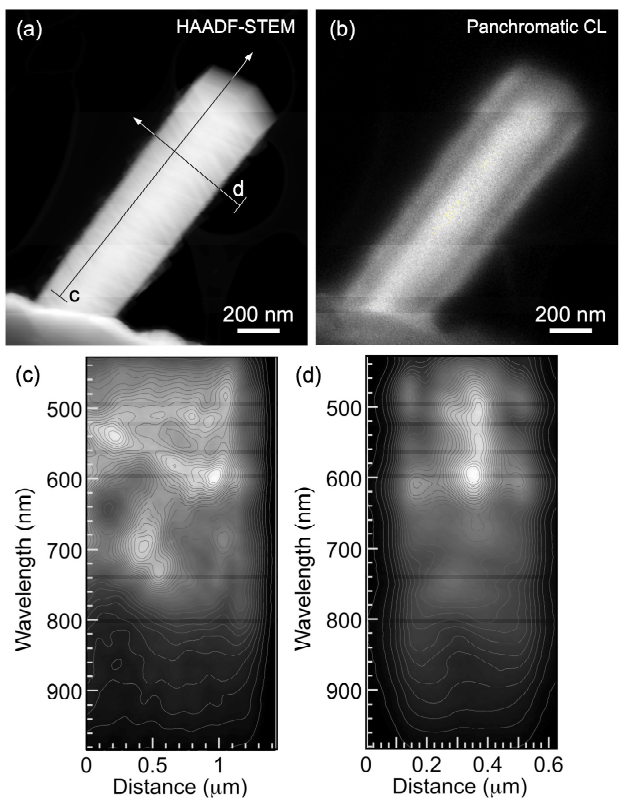

Figure S7. STEM-CL mapping and hyperspectral linescan of a thicker hexagonal GaS nanowire. (a) HAADF-STEM image of a thicker GaS nanowire with hexagonal cross-section. The tip with the VLS catalyst was likely broken off during dry-transfer to the TEM grid. (b) Panchromatic STEM-CL map of the nanowire shown in (a). (c) STEM spectral linescan along the line marked "c" in panel (a). (d) STEM spectral linescan across the wire (line "d" in panel (a)).

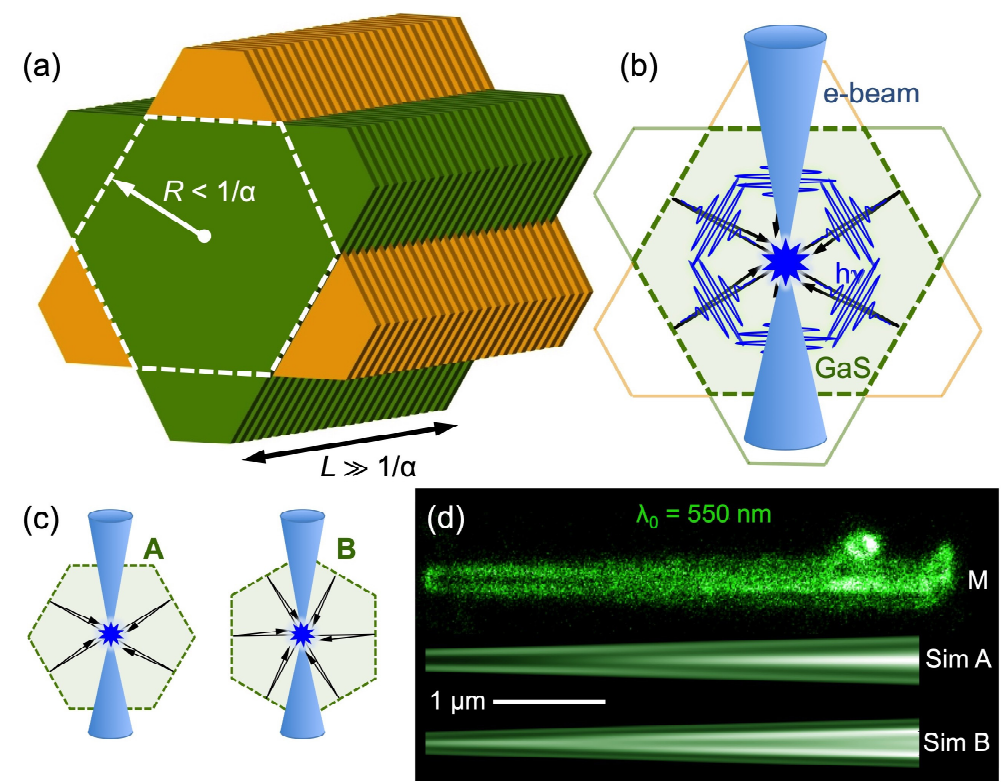

Figure S8. Interference of photonic waveguide modes launched by local electron beam excited luminescence. (a) Schematic of a type (ii) GaS nanowire with quasi-periodic twinning of truncated triangular few-layer flakes. Dashed line: outline of the hexagonal nanowire core. Note that the typical distances to the core surface $(R \leq 170 \mathrm{~nm})$ are smaller than the absorption length $\left(\alpha^{-1}\right)$ of $G a S$, whereas the distance to the end facets $L \gg \alpha^{-1}$, so that reflection of waveguide photons by the side facets yields interference while the modes traveling along the wire axis do not interfere, except near the ends of the wire. (b) Schematic of the electron beam excitation, primary luminescence, and backreflection by specular side facets. (c) Geometries of waveguide mode reflection for two different incidence directions of the electron beam. (d) Comparison of simulated interference patterns ('Sim A', 'Sim B', vacuum wavelength $\lambda_{0}=550 \mathrm{~nm}$ ) for geometries A and B shown in (c) with the experimentally measured intensity distribution ('M'). 


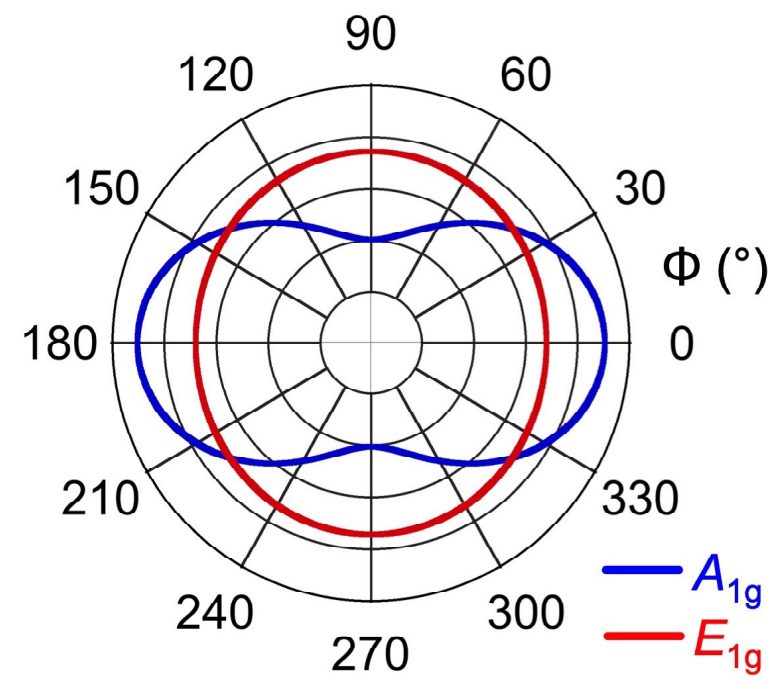

Figure S9. Predicted polarization-dependent Raman intensity for $A_{1 g}$ and $E_{1 g}$ modes of $G$ aS. Colored curves illustrate equations (3) and (4) in Supporting Note 2, respectively, which describe the experimental situation of polarized incident light but collection of all scattered light independent of polarization.

(a)
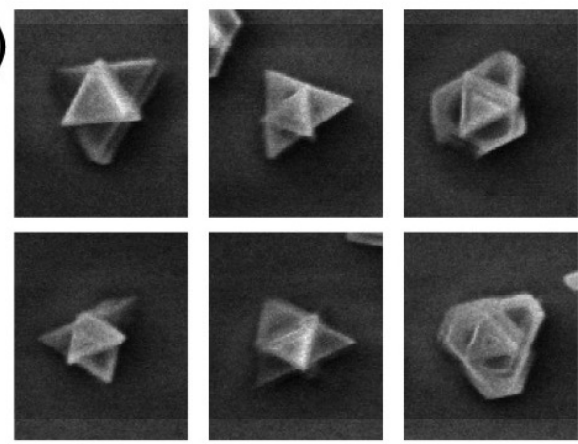

$1 \mu \mathrm{m}$ (b)

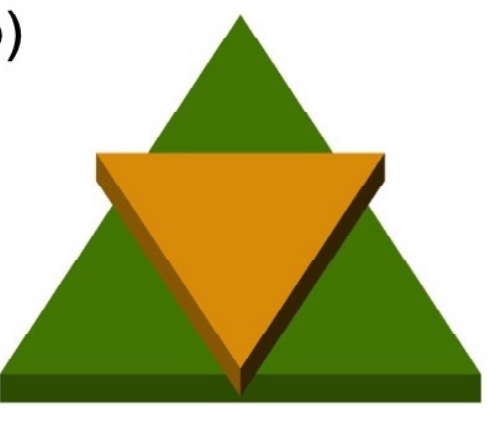

Figure S10. Twinning in planar GaS flakes. (a) Frequent twinning is observed in triangular and truncated triangular planar GaS flakes on the substrate surrounding fields of nanowires. (b) Schematic of the vertically stacked, twinned GaS flakes. 

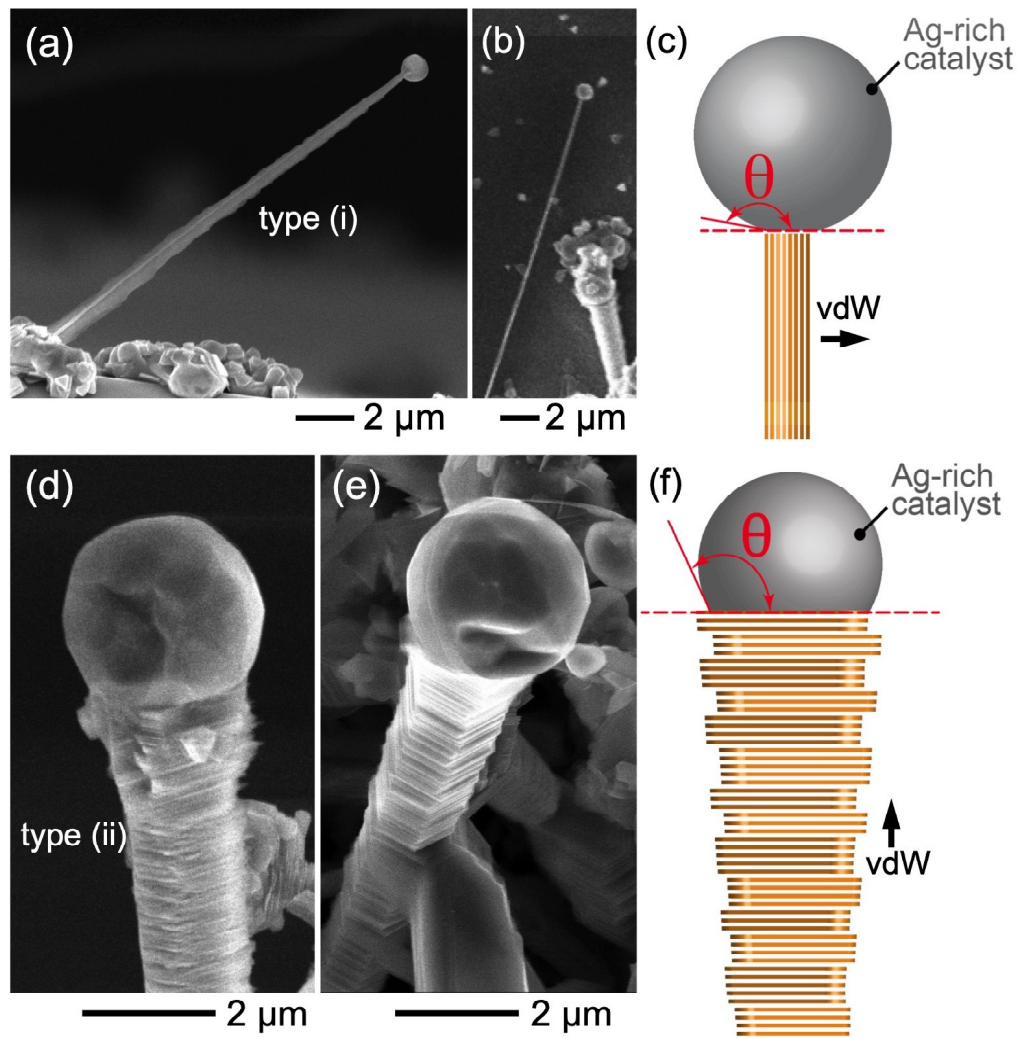

Figure S11. Differences in wetting of the Ag-rich VLS catalyst at the tips of type (i) and type (ii) GaS nanowires. (a) - (b) SEM image of a typical type (i) GaS nanowire. (c) Schematic illustration of the large contact angle $(\theta)$ of the Ag-rich catalyst at the type (i) nanowire tip, indicating non-wetting of the exposed GaS layer edges by the large catalyst drop. (d) - (e) SEM image of a typical type (ii) GaS nanowire. (f) Schematic illustration of the reduced contact angle $(\theta)$ of the Ag-rich catalyst at the type (ii) nanowire tip, indicating enhanced wetting of the GaS basal plane by the catalyst compared to type (i) nanowires.

\section{Supporting Notes}

\section{Supporting Note 1: Volume and Composition Evolution of the Ag VLS catalyst}

We used a geometrical analysis of tapered type (ii) wires to estimate the evolution of the $\mathrm{Ag}$ VLS catalyst during GaS nanowire growth. The spherical-cap shape of the catalyst is assumed constant during growth, and equal to the shape of the crystallized VLS tips observed by SEM (Figure 3 (a)). The corresponding catalyst drop volume is calculated as shown in Figure S6, for a base diameter of the spherical cap matching the local diameter of the tapered nanowire shown in Figure S6 (d). Using this procedure, we estimate that the volume of the VLS catalyst expands about 24-fold during the growth of the tapered type (ii) wire shown in Figure S6 (d).

This volume expansion can then be linked to a progressive increase in Ga content within the catalyst, assuming that the entire volume increase is due to retention of $\mathrm{Ga}$ in the drop (as described in the text). For this composition analysis, we assume that the initial composition is equal to that given by the Ag-Ga equilibrium phase diagram at the growth temperature $\mathrm{T}_{\mathrm{s}}=$ $600^{\circ} \mathrm{C}$, i.e., about 30 at. \% Ga (see Figure S6 (a)). The calculated volume increase then suggests a progressive increase in the Ga content to about 90 at. \% Ga at the end of the growth of the nanowire shown in Figure S6 (d). 


\section{Supporting Note 2: Polarized Raman Spectroscopy}

The analysis of the polarization-dependent Raman spectra follows the classification of the Raman-active modes of $\beta$-GaS by van der Ziel. ${ }^{1}$ For the Raman-active modes analyzed here, $E_{1 g}^{1}$ and $A_{1 g}^{1}$, the scattering tensor has the following non-vanishing components: $A_{1 g}^{1}: z z, x x+$ $y y$; and $E_{1 g}^{1}: z x, y z$. Hence, the scattering tensors can be written as follows:

$$
\mathrm{R}\left(A_{1 g}^{1}\right)=\left(\begin{array}{ccc}
a & 0 & 0 \\
0 & a & 0 \\
0 & 0 & b
\end{array}\right) ; \quad \mathrm{R}_{1}\left(E_{1 g}^{1}\right)=\left(\begin{array}{ccc}
0 & 0 & a \\
0 & 0 & 0 \\
a & 0 & 0
\end{array}\right), \quad \mathrm{R}_{2}\left(E_{1 g}^{1}\right)=\left(\begin{array}{ccc}
0 & 0 & 0 \\
0 & 0 & b \\
0 & b & 0
\end{array}\right) .
$$

For incident and scattered electric field (unit) vectors $\mathbf{e}_{\mathrm{i}}$ and $\mathbf{e}_{\mathrm{s}}$, respectively, the scattered intensity is calculated as:

$$
I=\left|\mathbf{e}_{s}^{T} \mathbf{R e}_{i}\right|^{2}
$$

where the superscript $\mathrm{T}$ denotes the transposition from a column vector to a row vector. ${ }^{2}$

If, as in our experiments, no analyzer is placed in the path of the scattered light, this relation simplifies to:

$$
I=\left|R \mathbf{e}_{i}\right|^{2}
$$

In our measurements on type (ii) GaS nanowires, the incident electric field vector lies in the $(\mathrm{x}, \mathrm{z})$ plane: $\mathbf{e}_{i}=\left(\begin{array}{c}\cos (\varphi) \\ 0 \\ \sin (\varphi)\end{array}\right)$.

For the $A_{1 g}^{1}$ mode: $\quad I=a^{2} \cos ^{2}(\varphi)+b^{2} \sin ^{2}(\varphi)$

For the $E_{1 g}^{1}$ mode: $\quad I=a^{2}+\frac{b^{2}}{2}(1-\cos (2 \varphi))$

Figure S9 illustrates the expected two-fold symmetry of polar plots of Raman intensity as a function of polarization angle $\varphi$ (compare also Fig. 5 of the main text):

\section{Supporting Note 3: Confined Photonic Waveguide Modes in GaS Nanowires}

A comparison of the measured monochromatic CL maps for three vacuum wavelengths $\lambda_{0}$ $(450,550$, and $650 \mathrm{~nm}$, shown in Figure 7 (f)) with simulations of the expected interference fringe pattern according to the model developed in Ref. 3 is used to establish interference of confined photonic waveguide modes as the origin of the observed intensity modulation in the experimental maps.

Waveguide modes are traveling photonic modes that propagate within the GaS nanowire (the 'waveguide'), with wavelength that is related to the vacuum wavelength $\lambda_{0}$ via $\lambda^{\mathrm{wg}}=\lambda_{0} n^{-1}$, where $n$ denotes the (photon energy dependent) refractive index of GaS. We have shown previously that nanometer-scale locally excitated luminescence in STEM-CL can launch such modes, which for photon energies in the transparency region can travel over significant distances in mesoscale structures. Specular surface facets, realized previously in mesoscale $\mathrm{GeS}_{\text {prisms }}{ }^{3}$ and here in type (ii) GaS nanowires, internally reflect the propagating modes so that they can coherently add and generate an interference pattern. Within this framework, constructive interference is expected whenever the excitation (i.e., the electron beam) is positioned at integer multiples of $\lambda^{\mathrm{wg}} / 2$ from the reflecting edge.

We have simulated the expected interference fringe pattern for the three vacuum wavelengths of our experimental monochromatic CL maps using the software package Mathematica. The excitation was placed at different distances from the (projected) edges of a type (ii) nanowire with the experimentally observed geometry (Figure 7 (a)). The overall intensity of the emitted light for each position of the exciting electron beam was computed by coherently adding the primary emission with beams internally reflected from the six sides of the hexagonal envelope 
of the nanowire (i.e., ignoring the corrugation of the protruding GaS flakes, see Figure S8 (b). The total intensity, with contributions from the entire thickness of the nanowire traversed by the electron beam, was then plotted for each wavelength as a false color density plot (Figure 7 (f)). We repeated this procedure for two orientations of the nanowire relative to the electron beam, namely 'edge up' (orientation $\mathbf{A}$ in Figure S8 (c)) and 'corner up' (orientation $\mathbf{B}$ in Figure S8 (c)). The observed fringe pattern fits closely with the experimentally observed intensity modulations at each wavelength if we assume the 'edge up' orientation (A) of the wire. The alternative 'corner up' orientation $(\mathbf{B})$ produces a different fringe pattern that does not match the intensity distribution observed experimentally (Figure S8 (d)).

\section{Supporting References}

1. van der Ziel, J. P.; Meixner, A. E.; Kasper, H. M. Raman Scattering from $\beta$-GaS. Solid State Commun. 1973, 12, 1213-1215.

2. Saito, R.; Tatsumi, Y.; Huang, S.; Ling, X.; Dresselhaus, M. S. Raman Spectroscopy of Transition Metal Dichalcogenides. J. Phys.: Condens. Matter 2016, 28, 353002.

3. Sutter, P.; Argyropoulos, C.; Sutter, E. Germanium Sulfide Nano-Optics Probed by STEMCathodoluminescence Spectroscopy. Nano Lett. 2018, 18, 4576-4583. 